\title{
PTSD Symptoms, Vicarious Traumatization, and Burnout in Front Line Workers in the Homeless Sector
}

\author{
Jeannette Waegemakers Schiff ${ }^{1}\left[\right.$. Annette M. Lane $^{2}$
}

Received: 16 March 2017 / Accepted: 12 December 2018 / Published online: 25 January 2019

(c) Springer Science+Business Media, LLC, part of Springer Nature 2019

\begin{abstract}
Work with clients who have trauma-related problems is reported to lead to a constellation of reactions including vicarious traumatization, compassion fatigue, and burnout. Traumatic experiences are ubiquitous in the lives of homeless people, stemming from multiple life events prior to, and as a result of, experiencing homelessness. While most studies examine either burnout and vicarious traumatization or potential PTSD in people who work with those who are traumatized, they generally do not look at the possible co-existence of all three factors. They also do not explore if these factors indicate the extent to which burnout or vicarious traumatization may lead to PTSD symptoms or that the existence of PTSD symptoms may precipitate greater rates of burnout. In addition, there are no existing studies that provide a quantitative view of the characteristics of frontline workers in homeless services. In this study, we surveyed 472 individuals who work in frontline positions in homeless shelters in 23 different organizations. We found rates of burnout, vicarious traumatization and compassion satisfaction, comparable to workers in other social services organizations but found rates of PTSD symptoms to be at 33\% of the total population. This higher incidence of PTSD symptoms suggests that workers under-report traumatic stress when it is described as vicarious traumatization, that they specifically attribute this to client contact, and that vicarious traumatization is traumatic stress specifically attributed to job-related events.
\end{abstract}

Keywords Homelessness services $\cdot$ Burnout $\cdot$ PTSD $\cdot$ Traumatic stress $\cdot$ Frontline staff

\section{Introduction}

Homeless individuals experience multiple traumas, stemming from turbulent life events both prior to, and as a result of, becoming homeless. Their complex histories are replete with trauma, abuse, violence, addictions and/or mental health issues (Hopper et al. 2009). Resultantly, staff working with homeless individuals are constantly exposed both to those who are traumatized and to traumatic situations, which are very hard on the human psyche and may lead to what is variously referred to as vicarious traumatization or secondary traumatic stress (Baird and Kracen 2006). As with other occupations such as emergency responders and troops in war zones where workers are repeatedly exposed

Jeannette Waegemakers Schiff

schiff@ucalgary.ca

1 Faculty of Social Work, University of Calgary, Calgary, Canada

2 Faculty of Health Disciplines, Athabasca University, Athabasca, Canada to traumatic events (Berger et al. 2012; Pinto et al. 2015), the impact of helping traumatized homeless individuals can lead to the development of elevated rates of a combination of burnout, compassion fatigue, vicarious traumatization, and post-traumatic stress in those who work with the homeless.

The rise in homelessness in the U.S. and Canada has led to a corresponding increase in the size of the workforce providing support services to this population, currently estimated in the U.S. at 130,000 workers in homelessness services and an additional equal number in ancillary services (Mullen and Leginski 2010). There is, to date, little information on the educational and training and work backgrounds of those who work in this field (Olivet 2010). However, while a number of studies report on traumatic stress in mental health therapists (Baum 2015), social work clinicians (Cunningham 2003), critical care nurses (Burgess et al. 2010), mental health professionals (Finklestein et al. 2015), and domestic violence services providers (Kulkarni et al. 2013), studies that examine psychological and mental health demands on frontline workers in homelessness services is lacking. What makes a closer examination of this specific 
cohort compelling is that we need to better understand the impact on services providers of providing, on a continual basis, supports and services to people with significant histories of traumatic experiences. Furthermore, while there are some reports of this staff having little or no substantive training in the many aspects of working with vulnerable and traumatized people (Mullen and Leginski 2010; Olivet et al. 2010) this information relies primarily on qualitative reports from organizations in the homelessness sector. This absence of data on training and experience, in addition to lack of understanding of work-related stressors of this workforce, makes it difficult to know what supports are needed to mitigate the risk for work-related stress disorders.

Examination of the multiple factors of burnout, vicarious traumatization (also referred to as secondary traumatic stress), compassion fatigue, and trauma symptoms may provide indicators of what stressors staff face and what training and supports are necessary to address these issues. While most studies examine either burnout and secondary traumatic stress (STS) or PTSD, few look at the co-existence of all three and resultant implications. In this study, we measured reported burnout, STS, compassion fatigue and PTSD symptoms of 472 frontline workers in two major Canadian cities and explored if staff who have high levels of burnout are at greater risk for development of PTSD and raise the issue of those in homeless support services as being in a high-risk occupation.

Initial descriptions of burnout identified it as consisting of three dimensions: emotional exhaustion, depersonalization, and diminished feelings of personal accomplishment (Maslach et al. 2001). More recently, burnout has been refined to conceptualize it as a facet of professional quality of life that is comprised of two components: compassion satisfaction and compassion fatigue. Encompassed in compassion fatigue are two sub-components: burnout and secondary traumatic stress (Stamm 2010). Compassion satisfaction refers to positive feelings derived from doing one's work well. On the opposite end, compassion fatigue consists of two distinct components of negative emotional and behavioral responses to stress in the work environment: burnout and secondary traumatic stress (STS). Burnout is marked by frustration, anger, depression, and exhaustion, while STS consists of the intrusive emotional responses of work-related exposure to traumatized people. Similar to PTSD symptoms, STS effects include anxiety, fear, and intrusive reminders of the traumatic events, sleep difficulties and avoidance of reminders of the traumas (Bell et al. 2003; Van Hook and Rothenberg 2009). STS symptoms are similar to those described as components of vicarious traumatization, and often the terms are used interchangeably in the research literature (Baird and Kracen 2006). Some researchers suggest that vicarious traumatization concerns itself with alterations in personal cognitive schemas of counselors where they no longer see the world as safe, and issues of control, trust, intimacy and esteem are challenged. However, these may also appear as ancillary features of PTSD. On the other hand, STS encompasses a wider range of phenomena usually associated with traumatic stress and PTSD, such as heightened anxiety, sleep disturbances, depression, intrusive thoughts, and avoidant behaviors (Figley 1995, 2013). Some work suggests that both burnout and STS are highly correlated (Cieslak et al. 2014) and are often connected to the work environment (Melvin 2015). While many of the symptoms of STS are the same as those of PTSD, reports in the research literature of the result of experiencing direct trauma, such as workers assisting others in war or natural disaster has commonly been accepted as defining the difference between PTSD and STS. The DSM-5 has added as an additional cause of PTSD, exposure to aversive details or experiencing repeat exposure to traumatic event(s) (American Psychiatric Association 2013), which suggests that STS is actually traumatization as identified in PTSD criteria through this additional avenue. This present study also provided some insight on this relationship.

Many studies, of which we note only a representative sample, report higher levels of stress responses among social workers, mental health professionals, nurses, child and youth workers, and child welfare workers (Sprang et al. 2011). This body of research suggests that burnout is widespread and significant levels are experienced by $21-67 \%$ of mental health and social work professionals. However, differing methodologies contribute to and may be responsible for this reported wide range of prevalence (Morse et al. 2012). In contrast to burnout studies, research on workplace related PTSD has largely focused upon nurses, emergency responders and mental health counsellors (Regehr 2018). Estimates of the incidence of PTSD range from 7 to $44 \%$ (Mealer et al. 2014) with rates of those meeting PTSD criteria among mental health nurses at 14\%, and 17\% (Lee et al. 2015) and social workers at $22.5 \%$ (Regehr et al. 2015) and most often connected traumatic stress symptoms with identifiable specific work-related events.

Factors that mitigate development of burnout or trauma symptoms may stem from individual and organizational sources. Research often examines individual coping and resiliency and include perceptions, meanings, or implications of the traumatic event (Kulkarni et al. 2013), positive coping methods (Burgess et al. 2010; Wood et al. 2012), aggression management training (Lee et al. 2015), strong leadership support (Wood et al. 2012), and support from colleagues and family/friends (Lavoie et al. 2011). This attention to individual vulnerabilities pays insufficient attention to organizational responses to burnout. More importantly, many organizations lack sufficient awareness of trauma and trauma-informed care (Substance Abuse and Mental Health Service Administration 2014) and traumatic events which 
impact stress and potential PTSD responses (Regehr et al. 2004).

Given the vast literature on burnout and PTSD that has developed over the last two decades, what contribution does the present study make regarding burnout and traumatic stress in support workers in the homeless sector? This group of workers is rapidly becoming recognized as being exposed to trauma on a continual basis (Hopper et al. 2009), with little training and sparse organizational supports for their work (Mullen and Leginski 2010; Olivet et al. 2010). These conditions indicate the strong probability of worker exposure to both burnout and traumatic stress as they work with homeless people, the vast majority of whom report trauma both prior to loss of housing and while experiencing homelessness (Mullen and Leginski 2010). This in turn leads to several questions that this study sought to answer: (1)What are the educational and training profiles of workers in frontline services in the homeless sector? (2) Is burnout or traumatic stress the most predominant stress factor in this workforce? (3) Is burnout or STS a greater predictor of PTSD symptoms? (4) Are there organizational factors that are correlated with burnout, STS or PTSD symptoms? Additionally, as we describe below, the inclusion of a direct measure of PTSD symptoms led us to look closely at this relationship to STS and burnout.

\section{Methodology}

The umbrella organizations which coordinate services for homeless services in two major Canadian cities identified major sector agencies, shelters, day, and support programs in each region. These agencies were then approached about participating in this study. This yielded a pool of 26 organizations, of which 23 participated, 10 in one city and 13 on the other. Using the approach described below, 499 surveys were distributed and 472 usable responses were collected for a response rate of $96 \%$. With consent of each participating agency, researchers provided preliminary information to senior management and subsequently attended staff meetings during which the study was explained and surveys distributed. Staff was provided time out during the staff meeting to retire to a private place to complete surveys, which were then returned in a sealed envelope. This approach had several advantages: it allowed for the least staff burden because they did not have to find additional time for survey completion; participants could decline (return blank copies); and it facilitated rapid response and a very high response rate. Overall, 18 surveys (less than $5 \%$ of the total) were not completed. This signified that some staff did exercise a choice not to participate. It also ensured that we had a high rate of participation by front-line workers as greater than $85 \%$ attended the meetings. Most of those absent were due to illness, vacation or client emergencies. We also had only one wave of data collection and thus minimized any possible response influence among peers who completed the survey. Thus, we were assured that the results are representative of the workforce in the homeless sector in these cities. The study was approved by the University of Calgary Ethics Boards.

The survey included demographics, items about job assignment, length of employment, employment status (full and part-time), educational background, job assignment, organizational mandate and philosophy (housing, harmreduction and, abstinence-based), supervision and teamwork that research suggests may be related to worker burnout. It also incorporated two well-established instruments, the PROQoL (Stamm 2010), which measures compassion satisfaction, compassion fatigue, and burnout, and the PCL (Wilkins et al. 2011) which assesses PTSD symptoms.

The PROQoL, widely used to assess the quality of professional life of people who work with those experiencing extremely stressful events (Stamm 2010), consists of 30 variables (5 point Likert scale) on three scales: compassion satisfaction, compassion fatigue and burnout. It has strong construct validity and scales have internal validity and consistency (compassion satisfaction 0.87, compassion fatigue 0.80, and burnout 0.72) (Bride et al. 2007). The PCL-C, (PTSD Check List), is used to assess PTSD symptoms in military and civilian populations (Wilkins et al. 2011). A short, six item version has been found to have strong sensitivity (0.92) and specificity (0.72) (Lang et al. 2012) and can be used as a reliable screen for traumatic stress and potential PTSD. The PCL- 6 short version was chosen because of its strong psychometric properties, relevance to a civilian population and a reduced burden of administration.

\section{Data Analysis}

We used SPSS-24 for statistical analyses, with cases of missing data excluded from the results. Some descriptive variables were collapsed into encompassing categories such as "support worker and case manager". To ensure that the data collected was a reliable indicator of the stressors reported we analyzed and compared scale properties of the PCL and PROQoL as reported in respective previous psychometric studies of each. The PCL had a Cronbach's alpha of 0.835, which supports what was reported previously (Wilkins et al. 2011). The PROQoL scales had Cronbach's alpha of 0.738 (burnout), 0.828 (STS vicarious traumatization) scale, and 0.884 (compassion satisfaction), all of which concur with norms reported by others (Bride et al. 2007; Stamm 2009).

The survey contained a number of items on workplace practices, including supervision, use of teams, and availability of peer support. In order to understand factors that may contribute to the PROQoL and PCL scores we conducted 
further data reduction through a factor analysis on the questions related to worker and managerial job-related behaviors such as adequate, regular administrative and clinical supervision, peer support by workers, debriefing of critical incidents and similar items. This resulted in three acceptable scales, termed peer support ( $\mathrm{N}=5$, alpha 0.826$)$, administrative support $(\mathrm{N}=6$, alpha 0.874$)$ and health support $(\mathrm{n}=6$, alpha 0.784 ), that were sufficiently strong and long enough to include in further analysis. However, cumulatively, they represent only $36 \%$ of the total variance. Thus though they are strong and may enlighten the understanding of the relationships among peer, administrative, and health supports in impacting traumatic stress and STS, these scales are insufficient to stand alone as explanatory for the PCL and PROQoL results.

\section{Results}

Description of the demographic profile is critically important as there are few, if any, reports of the composition of this work force, their background, and training needs. Demographic information focussed on the nature of the employment status, level of job preparation, and income of respondents. Some organizations have few staff (six to ten) and because of the perceived sensitive nature of many questions, the research team opted to exclude age and gender as potential identifiers as role, education, and training were important variables that would otherwise reduce anonymity. While this has some impact on results, it does not lessen the overall importance of the study. Over half of the respondents (51\%) report no more than 2 years of post-secondary education, and of this cohort, $25 \%$ had a 2-year diploma. A further $35 \%$ has a BA or equivalent, although the areas of study spanned most of the fields found in a liberal arts institution and were not concentrated on professional social services or health care education. Only 13\% report having a graduate degree. In many fields that train people to work in human services, a BA provides basic training and a graduate degree, specialized additional skills. The Council on Accreditation (2018) recommends a minimum level of a BA plus experience for supervisory positions. These minimal requirements are not the norm in organizations serving homeless individuals (Mullen and Leginski 2010). In this cohort, many lack adequate educational preparation for their jobs. This information is valuable as there are currently no educational or training requirements in homeless serving organizations (Mullen and Leginski 2010) and there are few colleges or universities that offer courses relevant to work in this field (Olivet et al. 2010).

Twenty-five percent of participants report being employed in the homeless sector for less than 1 year. This figure is significantly higher than the employment turnover rate of
17-19\% reported for non-profit organizations in both Canada and the US (Nonprofit 2017). Many staff (43\%) had been employed in the homeless sector for less than 2 years, and almost three-quarters (74\%) for less than 5 years. More importantly, $79 \%$ of support counselors and $77 \%$ of shelter staff had been employed in the homeless sector for less than 5 years and of these, $44 \%$ of support counselors and $53 \%$ of shelter staff had been in the sector less than 2 years. These low levels of experienced staff and their turn-over are alarming as there are few training opportunities outside of on the job sessions (Mullen and Leginski 2010) that would help them to gain the requisite knowledge base and skills for the work demands with highly traumatized and vulnerable people. Furthermore, young, inexperienced staff are rapidly promoted, even when their experience is limited, and thus their ability to supervise and manage is not well grounded. Neither level of education or background training had a significant relationship to the length of time a person had been employed in the sector. Many had also worked in the sector for less than a year $(25 \%)$ and the majority had worked in the sector for less than 5 years (74\%). Only $11 \%$ had been employed in the homeless sector for more than 10 years, even though most of the participating organizations have been in existence longer than that. Thus, this workforce is relatively untrained, and experiences rapid turn-over.

Participants represented a wide range of front-line roles, over half (53.9\%) reporting primary roles as intake, support counselor or shelter/emergency residence staff, clinical roles including case managers (17\%), and 8\% reported administrative and facilities responsibilities. Many (38\%) had multiple roles including administrative, direct services functions and "other" (teachers, nurses, child care workers and students). Most respondents (71\%) have total earnings of less than $\$ 50,000$ annually. The length of time that a person had been employed in the homeless sector was modestly (but significantly) correlated (0.407) with income, as was their highest achieved educational level (0.346), but only slightly $(0.131)$ with the stated primary role. In other words, people were likely to earn more over time and this was also dependent, but less so, on their educational achievement, and was not reflected in what kind of job role they had.

On all three scales of the PROQoL, the mean was 50 (in both cities) indicating average levels of burnout, vicarious traumatization and compassion satisfaction compared to others in helping professions (Stamm 2009). Importantly, 24\% of all respondents had burnout and vicarious traumatization at levels sufficiently elevated (57) to indicate that they should step back from their present responsibilities. Another $20 \%$ had a seriously diminished sense of compassion satisfaction, that is they no longer derive any innate positive feelings from helping others and may indeed be more negative than is therapeutically helpful to those in need. The scores on the PROQoL and PCL scales are reported in Table 1. 
Table 1 Scores on the PROQoL and PCL scales

\begin{tabular}{lcccc}
\hline & STS N=413 & Burnout N=440 & $\begin{array}{l}\text { Compassion Satisfac- } \\
\text { tion N=428 }\end{array}$ & PCL N=429 \\
\hline Mean & 50.0000 & 50.0000 & 50.0000 & 12.4143 \\
Std. Deviation & 9.98855 & 9.98860 & 9.98888 & 4.70612 \\
Range & $29.94-52.63$ & $26.15-89.33$ & $11.10-57.58$ & $6-24.00$ \\
Percentiles & & & & \\
25 & 42.4259 & 43.1977 & 43.7466 & 9.0000 \\
50 & 49.6307 & 49.4178 & 50.1032 & 12.0000 \\
75 & 56.2184 & 56.3718 & 57.5332 & 15.5000 \\
\hline
\end{tabular}

Table 2 Correlations of burnout, STS, traumatic stress and work variables

\begin{tabular}{|c|c|c|c|c|c|c|c|c|c|c|}
\hline Variable & $\mathrm{N}$ & 1 & 2 & 3 & 4 & 5 & 6 & 7 & 8 & 9 \\
\hline 1. Burnout & 440 & 1 & $0.594 * *$ & $-0.502 * *$ & $0.580 * *$ & $-0.272 * *$ & $-0.269 * *$ & $-0.283 * *$ & -0.056 & $0.111^{*}$ \\
\hline 2. STS & 413 & & 1 & $-0.254 * *$ & $0.611 * *$ & -0.067 & -0.063 & $-0.127 * *$ & -0.064 & 0.041 \\
\hline 3. Satisfaction & 428 & & & 1 & $-0.288 * *$ & $0.329 * *$ & $0.334 * *$ & $0.265^{* *}$ & -0.014 & 0.025 \\
\hline 4. PCL & 429 & & & & 1 & $-0.273 * *$ & $-0.165^{* *}$ & $-0.296 * *$ & -0.021 & 0.072 \\
\hline 5. Mgmt. Support & 413 & & & & & 1 & $0.639 * *$ & $0.532 * *$ & 0.029 & $-0.116^{*}$ \\
\hline 6. Peer Support & 417 & & & & & & 1 & $0.384 * *$ & -0.045 & $-0.128 * *$ \\
\hline 7. Health Support & 414 & & & & & & & 1 & 0.039 & -0.036 \\
\hline 8. Role & 426 & & & & & & & & 1 & 0.078 \\
\hline 9. Length of employment & 440 & & & & & & & & & 1 \\
\hline
\end{tabular}

*Correlation is significant at the 0.05 level (2-tailed)

**Correlation is significant at the 0.01 level (2-tailed)

The PCL has a 95\% accuracy rate for predicting PTSD based on scores of 14 or greater, Wilkins et al. (2011) report possible over-inflation of PTSD rates as there are no anchor points to a specific trauma, and the PCL may be overly sensitive to non-traumatic stressors. This caution is less important in light of the revised DSM-5 criteria for PTSD which no longer demand a specific event. On the other hand there is a distinct possibility of under reports of traumatic stress (Nishith et al. 2000) due to denial of severity of symptoms and social stigma that workers may feel (Regehr 2018). As the screen for PTSD, the PCL has been demonstrated to be both valid and highly reliable, and there is no longer the precise requirement for the identification of a precipitating traumatic event, present outcomes can be regarded as substantive indicators of high levels trauma induced anxiety and stress indicative of potential PTSD.

An analysis of the correlation between burnout, compassion satisfaction, secondary traumatic stress, PTSD symptoms with staff education, length of time of employment in the homeless sector, organizational and managerial support, showed small relationships among these variables (Table 2). We only report those with a correlation of greater than 0.250 $(\mathrm{p}<.001)$, that offer contributions and have some predictive ability. Some correlations have been reported in other studies and are understandable, such as that of the PCL and burnout
Table 3 Two-step regression analysis of the burnout, secondary traumatic stress (STS), and PCL scores

\begin{tabular}{lllllll}
\hline Model & \multicolumn{2}{l}{$\begin{array}{l}\text { Unstandardized } \\
\text { Coefficients }\end{array}$} & & $\begin{array}{l}\text { Standardized } \\
\text { Coefficients }\end{array}$ & t & Sig. \\
& \cline { 2 - 3 } & $\mathrm{B}$ & Std. Error & Beta & & \\
\hline 1 & & & & -1.968 & 0.050 \\
(Constant) & -1.898 & 0.964 & & 15.229 & 0.000 \\
STS & 0.286 & 0.019 & 0.611 & & \\
2 & & & & -4.894 & 0.000 \\
(Constant) & -4.959 & 1.013 & & 8.850 & 0.000 \\
STS & 0.196 & 0.022 & 0.417 & 6.906 & 0.000 \\
Burnout & 0.152 & 0.022 & 0.325 & &
\end{tabular}

Data tables CMHJ (2018)

Dependent Variable: PCL

$(0.580 ; \mathrm{p}<.000)$ and STS $(0.611, \mathrm{p}<.000)$. Compassion satisfaction was directly related to support from peers $(0.329$; $\mathrm{p}<0.000)$ and management $(0.334 ; \mathrm{p}<0.000)$. Not surprisingly, the compassion satisfaction scale has a small and negative $(-0.288)$ correlation with the PCL $(p<0.000)$, indicating that those with higher trauma symptoms had less satisfaction from helping others. A stepwise regression analysis (Table 3) of the PCL, burnout, and STS indicated a 
strong and meaningful relationship between STS and PTSD ( $\beta$ 0.611), suggesting that workers who experience vicarious traumatization are at greater risk for developing PTSD with increased exposure to trauma. While burnout also increases the risk for increased traumatic stress response, it is not as great $(\beta 0.325)$.

We also performed a stepwise hierarchical regression analysis with PCL score as the dependent variable and burnout and STS as the predictor variables. (Table 3) Model 1 with STS as the sole predictor produced an R of $0.612\left(R^{2}=0.374\right)$ and with both STS and burnout the result was $\mathrm{R}=.668\left(\mathrm{R}^{2}=446\right)$. An ANOVA of both models (Model 1: $\mathrm{F}=238.99, d f=1, \beta=0.286, \mathrm{p}, .000$, and Model 2: $d f=2, \mathrm{~F}=160.42, \beta(\mathrm{STS})=0.194, \mathrm{p} 0.000$, and $\beta$ (burnout $)=0.156, p 0.000$ ), were both highly significant $(\mathrm{p}<0.000)$. Thus, while both burnout and secondary traumatic stress may predispose a worker to PTSD symptoms (elevated PCL scores), the STS is a greater contributor than burnout. This is inherently reasonable as STS symptoms of intrusion, hypervigilance, and over-reactivity are largely similar to PTSD symptoms, while characteristics of burnout more often reflect anger, lack of caring and fatigue.

Lower rates and levels of burnout may be linked to clinical support from supervisors and managers (Kalliath and Beck 2001) and therefore, one original hypothesis was that managerial support would temper front-line workers experience of burnout. Correlations between each support scale and the PCL, burnout, and STS scales found mild but significant $(0.000$ level) relationships with a negative correlation of -0.272 between support and burnout and -0.273 between support and PTSD symptoms (PCL scores), suggesting that managerial and peer support had a tempering effect on traumatic symptoms. However, STS had no significant correlations with support or peer support, suggesting that vicarious traumatization, unlike compassion satisfaction, operates with some independence from supervisory and peer support.

An examination of the relationships between health supports, PCL scores, burnout and vicarious traumatization, revealed smaller but still significant $(\mathrm{p}>0.000)$ relationships among these variables. Health supports also attenuate the impacts of traumatic symptoms $(\beta-0.302)$, although at lower levels than supervisory and peer supports. One explanatory factor is that all workers are covered under universal health care that covers all hospital and outpatient physician's care. Ancillary care (dental, some physiotherapy, massage therapy, prescription medications) are provided under private plans that vary in their benefits. Thus, the lower levels may reflect the lessened impact of health costs to staff, but this remains speculative. There were also no significant relationships between income, length of employment in the homeless sector, and primary job role in these respondents. Although many have annual incomes below $\$ 50,000$ and have been employed in the sector for relatively short periods of time, these variables were only very slightly correlated with trauma or burnout and also had no impact on reported compassion satisfaction. Despite the widely accepted fact that workers are not paid well, they continue to care, and this caring does not, for most, diminish over time.

\section{Discussion}

The initial aim of this study was to examine the psychosocial stressors of frontline workers in the homelessness serving sector, with the hypothesis that reported high rates of traumatization by clients (Whitbeck et al. 2015) correspond with high emotional burden for staff (Olivet et al. 2010). Although burnout was hypothesized to be significant among this workforce, the unanticipated but most important result from this study was the high rate (33\%) of traumatic stress reported by frontline workers (PCL score of 14 or more). While the PCL is not a diagnostic instrument, as a preliminary screening for PTSD it is widely considered to be a strong indicator of its presence and an incidence of this magnitude (one-third of the participants) in this population is note-worthy. Additionally, symptoms of traumatic stress may be under-reported in staff as anecdotal reports from frontline staff indicate that this may be seen as a sign of personal weakness and an indicator of lack of suitability for work with homeless people. This observation of denial of stress because of its stigma has also been reported by others (Regehr 2018), When we include those individuals who fall one point below the cut-off score, this rate rises to $45 \%$ of all respondents. As staff report pressure to continue to work and because other research shows that there is more likely to be an under report of traumatic stress (Nishith et al. 2000), these high rates warrant serious attention.

This incidence of PTSD symptoms differs from other studies that found lower rates in frontline staff providing emergency services. A review of 28 studies of emergency workers by Berger et al. (Berger et al. 2012) reported rates of PTSD among police, firefighters and ambulance drivers across 14 countries on all continents, which ranged from 10 to $14 \%$. Further work by Pinto et al. (Pinto et al. 2015) indicates that perceived threat from the traumatic events in firefighters and emergency responders is a significant contributor to the onset of PTSD symptoms. Reported rates of traumatic stress and PTSD in nurses ranges from 14 to $17 \%$ (Lee et al. 2015). A recent study by Carleton et al. (2018) reported high rates of mental health symptoms clusters, including PTSD, among public safety personnel in Canada. However, as this study consisted of a web-based survey, the sample was self-selected and it is unknown what the actual sample size and response rates were. In the present study, participation of the total population was high and response rate very high, lending support for the validity of 
our findings. Although these results need to be treated with some caution, nonetheless, they suggest that they are in line with high rates of mental health problems in frontline workers and may be an accurate indicator of PTSD stress.

The higher rates of reported traumatic symptoms in this cohort of workers in the homeless sector may stem from several factors: the tendency of those who have suffered adverse events such as childhood abuse, domestic violence, mental health problems or addictions to become a part of the helping professions (Stevens and Higgins 2002), and their resultant vulnerability to developing traumatic stress responses (McFadden et al. 2014). The human origin of trauma, especially in childhood, has been shown to potentially have a greater and /or more prolonged effect on victims (Cloitre et al. 2009). Additionally, unlike emergency workers who experience traumatic incidents usually do not have a prior relationship with victims, and have their exposure limited to short time intervals, frontline workers interact with clients experiencing trauma in helping relationships over extended periods of time. This increases the probabilities of their exposure to trauma perpetrated by others, and complex trauma histories, and not by accidents or natural events.

Studies of traumatic stress in social workers (Cunningham 2003; Regehr 2018), nurses (Beck 2011) and mental health workers (Baum 2015) most often focus on vicarious traumatization and burnout (McFadden et al. 2014). Some work has also examined the relationship of prior histories of trauma and negative life experiences to the development of vicarious traumatization (Stevens and Higgins 2002), and have found that development of vicarious traumatization is more likely as a result of a history of childhood trauma (McFadden et al. 2014). However, these reports, primarily focused on burnout and vicarious traumatization, often based on small and convenience samples, are inconsistent and thus inconclusive (Stevens and Higgins 2002). Reports that examine burnout and vicarious traumatization generally do not report on the relationship between vicarious traumatization and primary traumatic stress symptoms. One exception is a study of mental health workers in the Gaza region, in which Finklestein et al. (2015) use the PTSD Inventory (Solomon et al. 1993) and the Compassion Fatigue Questionnaire (Figley 1995), which is an earlier version of the PROQoL, report a higher correlation (0.80) between PTSD and STS. The mental health workers in that study workers were constantly exposed to war-related trauma which may account for this relationship.

In the present study, we had hypothesized high rates of burnout, based on anecdotal reports that workers were highly stressed. Findings that workers had average rates of burnout, when compared with other studies of human services workers (Stamm 2010), but much higher rates of PTSD symptoms than reported elsewhere, raises questions both about the discrepancies between field reports of burnout in the homeless sector and etiology not widely discussed in the research literature. Given the results, we also explored the relationships between PTSD symptoms (the PCL), vicarious traumatization, and burnout and found high and significant correlations (0.613), but also evidence that these are related but not identical constructs. The most notable is the similarities and differences between vicarious traumatization and PTSD. We speculate that workers, based on their educational and experience background, and not usually working in trauma-informed organizations (Substance Abuse and Mental Health Service Administration 2014) are generally not well versed in trauma terminology and symptom constellations. Resultantly, they may misidentify traumatic symptoms of arousal and detachment as burnout in casual conversations and may also find it more acceptable to refer to burnout as a common denominator among staff, than traumatic response which may connote either psychopathology or lack of fitness for the job.

The present study was a pilot study of issues not previously examined in staff serving homeless people. Resultantly, we tried to minimize responder burden and thus did not inquire into the past history of workers or recent exposure to traumatic events, primarily because our initial hypothesis, based on field reports, was that workers were experiencing high rates of burnout. Thus, we did not anticipate that traumatic stress was the predominant issue. The PCL-6 was included in order to determine if direct traumatic stress was a factor in staff experiences, and the high rates of reported trauma symptoms have led us to include this screen in future studies. It also raised the distinct possibility that vicarious traumatization is a component of PTSD and not a different construct. The identifiable distinction is that in vicarious trauma the precipitant is most often due to hearing repeated stories of traumatic experiences of others. This is now included as a DSM criteria of PTSD. Thus we made need to re-think our conceptualizations of workplace trauma as an distinct work hazard.

Possible causes of the high rates of PTSD form some of our preliminary hypotheses that need further exploration. As McLaughlin et al. (2013) report, it may be the type, and not the number of prior traumatic experiences, and it may be the more expanded ways of conceptualizing traumatic exposure that become mitigating factors in the future development of PTSD, with experiences of interpersonal violence and childhood trauma important contributors. This leads us to offer some tentative explanatory factors. Many support workers are not well trained but may have entered the field because previous psychosocial problems and traumatic past experiences have led to a desire to help others, a phenomenon often seen in those recovering from addictions and in other areas of the helping professions (McFadden et al. 2014). Additionally, informal reports indicate that previously homeless persons enter this workforce as peer-informed staff. Research 
supports that their personal histories of childhood abuse, domestic violence, and addictions may have left them with prior and unresolved traumas that act as a catalyst for additional trauma experienced while working in the homeless sector (Brewin et al. 2000; Regehr 2018).

While trauma informed care has become a focus among services providers, it has been aimed at providing an understanding on trauma-based behaviors and appropriate interventions that workers can use in helping homeless persons and not at addressing trauma-related responses in the workforce staff. The present study suggests that there is a significant group of homeless support workers who are negatively impacted by their work, that this cohort is much larger than emergency responders such as police, EMTs and emergency room nurses, and that this constitutes both a workplace safety issue and an important intervention issue. We recognize that this study is a first step and its results indicate that further work is necessary to further explore the potential etiology and to quickly develop strategies to mitigate the consequences of trauma on those working in the homeless sector

While this study spanned two cities and 23 organizations and included 499 participants, it may be representative of western Canadian practices and not necessarily generalizable across front-line workers nation-wide or across North America. It is thus important to replicate the research and determine if these results are indeed indicative of systemic issues facing this specialized workforce. At the same time, the plight of those reporting high traumatic stress in this participant pool needs to also be addressed. We suggest that an intervention study be an additional priority, with the primary focus on organizational elements that can mitigate stress in this workforce along with implementing personal awareness and coping strategies that may increase personal self-efficacy and diminish the acuity of stress responses.

Funding Funding was provided by Calgary Homeless Foundation and Edmonton Homeward Trust.

\section{References}

American Psychiatric Association. (2013). Diagnostic and statistical manual of mental disorders (5th ed.). Washington DC.: American Psychiatric Association.

Baird, K., \& Kracen, A. C. (2006). Vicarious traumatization and secondary traumatic stress: A research synthesis*. Counselling Psychology Quarterly, 19(2), 181-188.

Baum, N. (2015). Secondary traumatization in mental health professionals a systematic review of gender findings. Trauma, Violence, \& Abuse, 17(2), 221-235.

Beck, C. T. (2011). Secondary traumatic stress in nurses: A systematic review. Archives of psychiatric nursing, 25(1), 1-10.
Bell, H., Kulkarni, S., \& Dalton, L. (2003). Organizational prevention of vicarious trauma. Families in Society: The Journal of Contemporary Social Services, 84(4), 463-470.

Berger, W., Coutinho, E. S. F., Figueira, I., Marques-Portella, C., Luz, M. P., Neylan, T. C., et al. (2012). Rescuers at risk: A systematic review and meta-regression analysis of the worldwide current prevalence and correlates of PTSD in rescue workers. Social Psychiatry and Psychiatric Epidemiology, 47(6), 1001-1011.

Brewin, C. R., Andrews, B., \& Valentine, J. D. (2000). Meta-analysis of risk factors for posttraumatic stress disorder in trauma-exposed adults. Journal of Consulting and Clinical Psychology, 68(5), 748.

Bride, B. E., Radey, M., \& Figley, C. R. (2007). Measuring compassion fatigue. Clinical Social Work Journal, 35(3), 155-163.

Burgess, L., Irvine, F., \& Wallymahmed, A. (2010). Personality, stress and coping in intensive care nurses: A descriptive exploratory study. Nursing in critical care, 15(3), 129-140.

Carleton, R. N., Afifi, T. O., Turner, S., Taillieu, T., Duranceau, S., LeBouthillier, D. M., et al. (2018). Mental disorder symptoms among public safety personnel in Canada. The Canadian Journal of Psychiatry, 63(1), 54-64.

Cieslak, R., Shoji, K., Douglas, A., Melville, E., Luszczynska, A., \& Benight, C. C. (2014). A meta-analysis of the relationship between job burnout and secondary traumatic stress among workers with indirect exposure to trauma. Psychological Services, 11(1), 75.

Cloitre, M., Stolbach, B., Herman, J., van der Kolk, B., Pynoos, R., Wang, J., \& Petkova, E. (2009). A Developmental approach to complex PTSD: Childhood and adult cumulative trauma as predictors of symptom complexity. Journal of Traumatic Stress., 79(1), $1-10$.

Council on Accreditation.(2018). http://coanet.org.

Cunningham, M. (2003). Impact of trauma work on social work clinicians: Empirical findings. Social work, 48(4), 451-459.

Figley, C. R. (1995). Compassion fatigue: Coping with secondary traumatic stress disorder in those who treat the traumatized. Abingdon: Routledge.

Figley, C. R. (2013). Compassion fatigue: Coping with secondary traumatic stress disorder in those who treat the traumatized: Abingdon: Routledge.

Finklestein, M., Stein, E., Greene, T., Bronstein, I., \& Solomon, Z. (2015). Posttraumatic stress disorder and vicarious trauma in mental health professionals. Health \& social work, 40(2), e25-e31.

Hopper, E. K., Bassuk, E. L., \& Olivet, J. (2009). Shelter from the storm: Trauma-informed care in homelessness services settings. The Open Health Services and Policy Journal., 2, 131-151.

Kalliath, T. J., \& Beck, A. (2001). Is the path to burnout and turnover paved by a lack of supervisory support? A structural equations test. New Zealand Journal of Psychology, 30(2), 72.

Kulkarni, S., Bell, H., Hartman, J. L., \& Herman-Smith, R. L. (2013). Exploring individual and organizational factors contributing to compassion satisfaction, secondary traumatic stress, and burnout in domestic violence service providers. Journal of the Society for Social Work \& Research, 4(2), 114-130.

Lang, A. J., Wilkins, K., Roy-Byrne, P. P., Golinelli, D., Chavira, D., Sherbourne, C., et al. (2012). Abbreviated PTSD Checklist (PCL) as a guide to clinical response. General Hospital Psychiatry, 34(4), 332-338.

Lavoie, S., Talbot, L., \& Mathieu, L. (2011). Post-traumatic stress disorder symptoms among emergency room nurses: Their perspective and a 'tailor-made' solution. Journal of Advanced Nursing, 67(7), 1514-1222. https://doi.org/10.1111/j.1365-2648.2010.05584.x.

Lee, J., Daffern, M., Ogloff, J. R., \& Martin, T. (2015). Towards a model for understanding the development of post-traumatic stress and general distress in mental health nurses. International journal of mental health nursing, 24(1), 49-58.

Maslach, C., Schaufeli, W. B., \& Leiter, M. P. (2001). Job burnout. Annual Review of Psychology, 52, 379-422. 
McFadden, P., Campbell, A., \& Taylor, B. (2014). Resilience and burnout in child protection social work: Individual and organisational themes from a systematic literature review. British Journal of Social Work, bct 210.

McLaughlin, K. A., Koenen, K. C., Hill, E. D., Petukhova, M., Sampson, N. A., Zaslavsky, A. M., \& Kessler, R. C. (2013). Trauma exposure and posttraumatic stress disorder in a national sample of adolescents. Journal of the American Academy of Child \& Adolescent Psychiatry, 52(8), 815-830. e814.

Mealer, M., Conrad, D., Evans, J., Jooste, K., Solyntjes, J., Rothbaum, B., \& Moss, M. (2014). Feasibility and acceptability of a resilience training program for intensive care unit nurses. American Journal of Critical Care, 23(6), e97-e105.

Melvin, C. S. (2015). Historical review in understanding burnout, professional compassion fatigue, and secondary traumatic stress disorder from a hospice and palliative nursing perspective. Journal of Hospice \& Palliative Nursing, 17(1), 66-72.

Morse, G., Salyers, M. P., Rollins, A. L., Monroe-DeVita, M., \& Pfahler, C. (2012). Burnout in mental health services: A review of the problem and its remediation. Administration and Policy in Mental Health and Mental Health Services Research, 39(5), 341-352.

Mullen, J., \& Leginski, W. (2010). Building the capacity of the homeless service workforce. Open Health Services and Policy Journal, $3,101-110$.

Nishith, P., Mechanic, M. B., \& Resick, P. A. (2000). Prior interpersonal trauma: the contribution to current PTSD symptoms in female rape victims. Journal of abnormal psychology, 109(1), 20.

Nonprofit, H. R. (2017). 2016 Nonprofit Emplyment Praqctices Survey. http://www.nonprofithr.com/wp-content/uploads/2016/04/2016N EPSurvey-final.pdf.

Olivet, J., McGraw, S., Grandin, M., \& Bassuk, E. (2010). Staffing challenges and strategies for organizations serving individuals who have experienced chronic homelessness. The Journal of Behavioral Health Services and Research, 37(2), 226-238.

Pinto, R. J., Henriques, S. P., Jongenelen, I., Carvalho, C., Maia, ÂC., Jongenelen, I., et al. (2015). The Strongest correlates of PTSD for firefighters: Number, recency, frequency, or perceived threat of traumatic events? Journal of traumatic stress, 28(5), 434-440. https://doi.org/10.1002/jts.22035.

Regehr, C. (2018). Stress, trauma, and decision-making for social workers. New York: Columbia University Press.

Regehr, C., Hemsworth, D., Leslie, B., Howe, P., \& Chau, S. (2004). Predictors of post-traumatic distress in child welfare workers: A linear structural equation model. Children and Youth Services Review, 26(4), 331-346.

Regehr, C., LeBlanc, V. R., Bogo, M., Paterson, J., \& Birze, A. (2015). Suicide risk assessments: Examining influences on clinicians' professional judgment. American Journal of Orthopsychiatry, 85(4), 295.

Solomon, Z., Benbenishty, R., Neria, Y., Abramowitz, M., Ginzburg, K., \& Ohry, A. (1993). Assessment of PTSD: Validation of the revised PTSD Inventory. Israel Journal of Psychiatry and Related Sciences, 30, 110-110.

Sprang, G., Craig, C., \& Clark, J. (2011). Secondary traumatic stress and burnout in child welfare workers: A comparative analysis of occupational distress across professional groups. Child Welfare, 90(6), 149-168.

Stamm, B. H. (2009). Professional quality of life: Compassion satisfaction and fatigue version 5 (ProQOL).

Stamm, B. H. (2010). The ProQOL manual: The professional quality of life scale compassion satisfaction (2 edn.). Pocatello: ProQOL. org. B Compassion Fatigue/Secondary Trauma Scales.

Stevens, M., \& Higgins, D. J. (2002). The influence of risk and protective factors on burnout experienced by those who work with maltreated children. Child Abuse Review, 11(5), 313-331. https ://doi.org/10.1002/car.754.

Substance Abuse and Mental Health Service Administration. (2014). TIP 54 Trauma-Informed Care in Behavioral Health Services Washington, DC: US Government. https://store.samhsa.gov/produ ct/TIP-57-Trauma-Informed-Care-in-Behavioral-Health-Services/ SMA14-4816.

Van Hook, M. P., \& Rothenberg, M. (2009). Quality of life and compassion satisfaction/fatigue and burnout in child welfare workers: A study of the child welfare workers in community based care organizations in central Florida. Social Work and Christianity, $36(1), 36$.

Whitbeck, L. B., Armenta, B. E., \& Gentzler, K. C. (2015). Homelessness-related traumatic events and PTSD among women experiencing episodes of homelessness in three U.S. Cities. Journal of traumatic stress, 28(4), 355-360. https://doi.org/10.1002/jts.22024.

Wilkins, K. C., Lang, A. J., \& Norman, S. B. (2011). Synthesis of the psychometric properties of the PTSD checklist (PCL) military, civilian, and specific versions. Depression and anxiety, 28(7), 596-606.

Wood, M. D., Foran, H. M., Britt, T. W., \& Wright, K. M. (2012). The impact of benefit finding and leadership on combat-related PTSD symptoms. Military Psychology, 24(6), 529.

Publisher's Note Springer Nature remains neutral with regard to jurisdictional claims in published maps and institutional affiliations. 